OPEN ACCESS

Edited by:

Christian Gaser,

Friedrich-Schiller-Universität Jena,

Germany

Reviewed by:

Svenja Caspers,

University Hospital Dusseldorf,

Germany

Xuntao Yin,

Army Medical University, China

*Correspondence:

Hua-Jun Chen

chj0075@126.com

tThese authors have contributed equally to this work

Specialty section

This article was submitted to

Applied Neuroimaging,

a section of the journal

Frontiers in Neurology

Received: 17 October 2018

Accepted: 10 January 2019

Published: 29 January 2019

Citation:

Zhan C, Chen H-J, Gao Y-Q and

Zou T-X (2019) Functional

Network-Based Statistics Reveal

Abnormal Resting-State Functional

Connectivity in Minimal Hepatic

Encephalopathy. Front. Neurol. 10:33.

doi: 10.3389/fneur.2019.00033

\section{Functional Network-Based Statistics Reveal Abnormal Resting-State Functional Connectivity in Minimal Hepatic Encephalopathy}

\author{
Chuanyin Zhan ${ }^{\dagger}$, Hua-Jun Chen ${ }^{*+}$, Yong-Qing Gao ${ }^{\dagger}$ and Tian-Xiu Zou \\ Department of Radiology, Fujian Medical University Union Hospital, Fuzhou, China
}

Purpose: Whole-brain functional network analysis is an emerging methodology for exploring the mechanisms underlying hepatic encephalopathy (HE). This study aimed to identify the brain subnetwork that is significantly altered within the functional connectome in minimal HE (MHE), the earliest stage of HE.

Materials and Methods: The study enrolled 19 cirrhotic patients with MHE and 19 controls who underwent the resting-state functional magnetic resonance imaging and cognitive assessment based on the Psychometric Hepatic Encephalopathy Score (PHES). A whole-brain functional connectivity (FC) matrix was calculated for each subject. Then, network-based statistical analyses of the functional connectome were used to perform group comparisons, and correlation analyses were conducted to identify the relationships between FC alterations and cognitive performance.

Results: MHE patients showed significant reduction of positive FC within a subnetwork that predominantly involved the regions of the default-mode network, such as the bilateral posterior cingulate gyrus, bilateral medial prefrontal cortex, bilateral hippocampus and parahippocampal gyrus, bilateral angular gyrus, and left lateral temporal cortex. Meanwhile, MHE patients showed significant reduction of negative FC between default-mode network regions (such as the bilateral posterior cingulate gyrus, medial prefrontal cortex, and angular gyrus) and the regions involved in the somatosensory network (i.e., bilateral precentral and postcentral gyri) and the language network (i.e., the bilateral Rolandic operculum). The correlations of FC within the default-mode subnetwork and PHES results were noted.

Conclusion: Default-mode network dysfunction may be one of the core issues in the pathophysiology of MHE. Our findings support the notion that HE is a neurological disease related to intrinsic brain network disruption.

Keywords: minimal hepatic encephalopathy, resting-state functional magnetic resonance imaging, brain functional network, cognitive impairment, network based statistic 


\section{INTRODUCTION}

Minimal hepatic encephalopathy (MHE) is a frequent cognitive complication of hepatic cirrhosis, diagnosed in up to $80 \%$ of patients with cirrhosis (1). It represents the mildest form of hepatic encephalopathy (HE) and is characterized by subtle cognitive impairments, but without the recognizable clinical symptoms of HE (2). In MHE, the cognitive abnormalities primarily involve deficits in speed of information processing, attention, executive control, motor ability, and coordination (3). MHE is associated with impaired quality of life and daily function (e.g., driving capability), as well as a higher risk of progression to overt HE (4-7). Recently, increasing attention has been devoted to uncovering the mechanisms underlying MHE, as understanding these mechanisms would be very helpful for the early diagnosis of MHE and prompt therapy, which can contribute to the improvement of prognosis $(1,7)$.

Whole-brain functional network analysis is an emerging methodology for the investigation of the pathophysiology of MHE. For example, based on whole-brain functional connectivity (FC) analysis at the region of interest (ROI)wise level, Zhang et al. (8) demonstrated widespread cortical and subcortical network connectivity alterations in MHE patients, particularly disrupted basal ganglia-thalamocortical FC and abnormal intracortical FC (predominantly in the form of decreased connectivity). In addition, based on wholebrain FC analysis at the voxel-wise level, previous studies outlined the whole-brain FC abnormalities (predominantly in the form of decreased connectivity) in cirrhotic patients with cognitive impairments, which are primarily distributed in the default-mode network (DMN), visual network, attention network, and thalamocortical loop $(9,10)$. Moreover, nearly all of the FC abnormalities mentioned above were found to be correlated with neuropsychological measurements, which indicates the important role of cortical and subcortical functional disorganization in the mechanisms underlying MHE. While the existing whole-brain functional network studies have helped expand our knowledge of the neural substrates underlying MHE, these studies have only examined whole-brain FC with a single seed region or via pairwise coupling. These approaches may fail to elucidate the role of the connections within the larger network; moreover, they do not provide insight into how MHE is related to the restructuring of functional networks, particularly at the connectome level.

A graph theory-based approach has been successfully used to perform functional connectome analysis by modeling the whole brain as a network, in investigations of organizational changes caused by MHE $(11,12)$. The graph theory-based analysis demonstrated that the topological organization of the functional whole-brain network in MHE patients tends to be more random, less clustered, and less modular (12, 13). Moreover, the topological properties of functional brain networks altered stepwise with the progression of $\operatorname{HE}(11,12)$.

Abbreviations: MHE, minimal hepatic encephalopathy; HE, hepatic encephalopathy; DMN, default-mode network; NBS, Network based statistic; PHES, Psychometric Hepatic Encephalopathy Score; FC, functional connectivity.
Another nonparametric statistical approach, called "networkbased statistics" (NBS) (14), has also been used in the field of connectome analysis. NBS is a method that controls the familywise error rate during the connectome analysis; it is a massunivariate testing method based on a network component, rather than an individual link. NBS has been validated as a method for localizing dysfunctional brain connectivity and applied in studies of several neuropsychiatric illnesses, such as Alzheimer's disease (15), Parkinson's disease (16), schizophrenia (17), autism (18), and major depression (19). The existing studies have demonstrated that NBS provides a general framework for the characterization of specific network components, which may be helpful for identifying which subnetworks, if any, exhibit significant functional differences in patients vs. healthy controls. Such an understanding of the FC alteration patterns in patients could aid in elucidating the neuropathological mechanisms underlying MHE. However, few whole-brain NBS studies have been conducted on MHE patients, which limits the further clarification of the network pathophysiology of MHE.

To address this issue, we conducted a comprehensive investigation of the whole-brain functional connectome in cirrhotic patients with MHE in order to identify the FC alteration patterns in MHE. Furthermore, we aimed to demonstrate the association between these changes in FC patterns and cognitive decline in MHE patients.

\section{MATERIALS AND METHODS}

\section{Participants}

This study was approved by the Research Ethics Committee of Fujian Medical University Union Hospital, China. Written informed consent was obtained from each participant prior to the study. Nineteen cirrhotic patients with MHE and 19 healthy controls were included. The demographic and clinical characteristics of all participants are listed in Table 1. There were no significant differences between the two groups in terms of age, gender, or education level. The Child-Pugh score was used to evaluate functional status of the liver for each patient. The Psychometric Hepatic Encephalopathy Score (PHES) examination-including the digit symbol test, number connection test $\mathrm{A}$, number connection test $\mathrm{B}$, the serial dotting test, and the line tracing test-was used for MHE diagnosis as described previously (9).

The exclusion criteria were as follows: (1) diagnosis of current overt HE or another neuropsychiatric disorder; (2) currently taking psychotropic medications; (3) diagnosis of uncontrolled endocrine or metabolic disease (e.g., thyroid dysfunction); (4) alcohol abuse within 6 months prior to the study; (5) contraindications for MRI.

\section{MRI Data Acquisition}

MR Imaging was performed using a $3.0 \mathrm{~T}$ scanner (Siemens, Verio, Germany). Three-dimensional T1-weighted magnetization-prepared rapid gradient echo (MPRAGE) sagittal images were collected with the following parameters: TR $=$ $1.9 \mathrm{~ms}, \mathrm{TE}=2.48 \mathrm{~ms}$, matrix $=256 \times 256, \mathrm{FOV}=256 \times$ $256 \mathrm{~mm}$, flip angle $=9^{\circ}$, slice thickness $=1.0 \mathrm{~mm}$ (without 
TABLE 1 | Demographic and clinical characteristics of the subjects.

\begin{tabular}{lccc}
\hline & $\begin{array}{c}\text { HC subjects } \\
(\boldsymbol{n}=\mathbf{1 9})\end{array}$ & $\begin{array}{c}\text { MHE patients } \\
(\boldsymbol{n}=\mathbf{1 9})\end{array}$ & $\boldsymbol{P}$-value \\
\hline Age (year) & $50.2 \pm 9.3$ & $50.2 \pm 9.4$ & 0.90 \\
Gender (Male/Female) & $16 / 3$ & $17 / 2$ & $0.63\left(\chi^{2}\right.$ test) \\
$\begin{array}{l}\text { Education level (year) } \\
\text { Etiology of cirrhosis }\end{array}$ & $8.7 \pm 1.8$ & $8.8 \pm 1.9$ & 0.30 \\
(HBV/alcoholism/ & - & $11 / 4 / 2 / 2$ & - \\
HBV+alcoholism/other) & & & \\
Child-Pugh stage (A/B/C) & - & $2 / 11 / 6$ & - \\
PHES TESTS & & & \\
Final PHES (score) & $0.5 \pm 1.0$ & $-7.8 \pm 3.4$ & $<0.001$ \\
$\begin{array}{l}\text { Number connection test A } \\
\text { (seconds) }\end{array}$ & $35.9 \pm 7.4$ & $59.4 \pm 20.6$ & $<0.001$ \\
$\begin{array}{l}\text { Number connection test B } \\
\text { (seconds) }\end{array}$ & $58.3 \pm 14.9$ & $128.3 \pm 62.0$ & $<0.001$ \\
$\begin{array}{l}\text { Serial dotting test (seconds) } \\
\text { Digit symbol test (raw score) }\end{array}$ & $42.5 \pm 6.1$ & $62.3 \pm 14.8$ & $<0.001$ \\
Line tracing test (raw score) & $112.7 \pm 18.8$ & $192.5 \pm 36.3$ & $<0.001$ \\
\hline
\end{tabular}

MHE, minimal hepatic encephalopathy; HC, healthy control; PHES, psychometric hepatic encephalopathy score; HBV, Hepatitis B Virus.

interslice gap), and 176 slices. Functional images were collected with an echo-planar imaging sequence using the following parameters: $\mathrm{TR}=2,000 \mathrm{~ms}$, $\mathrm{TE}=25 \mathrm{~ms}$, matrix $=64 \times 64$, FOV $=240 \times 240 \mathrm{~mm}$, flip angle $=90^{\circ}$, slice thickness $=4 \mathrm{~mm}$ (without interslice gap), 35 contiguous axial slices, and 180 volumes. Participants were instructed to keep their eyes closed, not think about anything specific, and remain still.

\section{Functional MRI Data Preprocessing}

SPM software and the Data Processing Assistant for RestingState fMRI tool (DPARSF 3.0, http://www.restfmri.net/forum/ DPARSF) were used to preprocess functional MRI data. The first 10 volumes were discarded to reduce the influence of the unstable initial MR imaging signal. Then, slice timing correction and realignment for head motion correction were performed. A subject was excluded if his/her translational movement was $>2.0 \mathrm{~mm}$ and the rotation was $>2.0^{\circ}(20,21)$. The individual T1-weighted images were coregistered to the mean functional images. A unified segmentation algorithm was then used to segment the transformed structural images into gray matter, white matter, and cerebrospinal fluid. Then, based on the normalization parameters estimated during unified segmentation, the motion-corrected functional images were further normalized to the standard Montreal Neurological Institute (MNI) space and resampled to $3 \times 3 \times 3 \mathrm{~mm}$. Subsequently, the functional images were spatially smoothed using a Gaussian kernel with $4 \mathrm{~mm}$ full width at half maximum and were linearly detrended. The resulting data were further bandpass-filtered $(0.01-0.08 \mathrm{~Hz})$ to reduce the low-frequency drift and high-frequency physiological respiratory and cardiac noise. To control for the possible effects of head motion and of the global white matter and cerebrospinal fluid signals on the results, we used linear regression to remove several sources of spurious variance, including six head motion parameters and the average signals from the cerebrospinal fluid, white matter, and whole brain.

\section{Network-Based Statistics}

In order to obtain a whole-brain FC matrix for each subject, we defined a set of 90 nodes across the brain based on the Automated Anatomical Labeling (AAL) atlas. For a given node region, the mean time course was calculated as the average of the fMRI time series from all voxels within that region. Then, the correlation matrices could be obtained by computing the Pearson correlation coefficient between the mean time course of each pair of nodes. Thereby, we were able to assess the FC differences between specific pairs of cerebral nodes. The network-based statistics (NBS) tool developed by Zalesky et al. (14) was used for the statistical comparison. Compared to the alternative methods for analyzing resting-state fMRI data (such as seed-based correlation approach and independent components analysis, ICA), NBS consider the whole brain as an integrated system (a connected network) rather than a collection of individual components (22). Thus, NBS allows the higher dimensional analysis rather than the low-dimensional ICA and seed-based correlation analysis, which can make a more comprehensive examination of network connections possible (23). Meanwhile, as a validated statistical method to deal with multiple comparisons during the whole-brain connectivity analysis, NBS can yield substantially greater statistical power than generic procedures, if any connected component (subnetwork) in which the connectivities show significant between-group difference exists (14). In other words, NBS could be more sensitive to identify which subnetwork, if any, exhibit significant functional differences between patients and controls. In this study, to compare the FC matrices of the two groups, a two-sample $t$-test was used with 5,000 permutations, and the significant $P<0.05$ was corrected for multiple comparisons using NBS correction (14). The significance was measured based on extent, not intensity. Notably, NBS controls for multiple comparisons through cluster-based thresholding, in which connected components of a network are considered a cluster. In this study, the primary cluster-defining threshold $(t$ $=3.1$ ) was used to identify a set of suprathreshold edges. Since NBS results are highly dependent on the initial cluster-defining threshold, we performed an additional series of NBS analyses with distinct initial cluster-defining thresholds $(t=2.8-3.4)$ as described in a previous study (24). Very similar results were obtained from these analyses (see Supplementary Figure 1); therefore, we only show the result of NBS analysis with the median cluster-defining threshold $(t=3.1)$ in this article.

\section{Correlation Analysis}

First, the strength of the FC within each subnetwork identified by NBS analysis was calculated. Then, a nonparametric approach, Spearman correlation analysis, was used to assess the association between patients' cognitive performance and FC strength. To correct for multiple comparisons, a false discovery rate (FDR) procedure was performed at $q<0.05$. 


\section{RESULTS}

MHE patients performed significantly worse on all PHES subtests and therefore had lower PHES scores than controls. This result indicates significant cognitive dysfunction in MHE patients.

NBS analysis identified two subnetworks with significant differences in FC matrices between patients and controls. The first subnetwork consisted of 15 nodes and 22 edges, with the edges representing a positive functional correlation among the nodes in the controls (Figure 1 and Table 2). The second subnetwork consisted of 14 nodes and 21 edges; in this subnetwork, the edges represented a negative functional correlation among the nodes in the controls (Figure 2 and Table 3).

Compared to controls, MHE patients showed a significant reduction of positive FC within the first subnetwork (Figure 1 and Table 2). This subnetwork predominantly involved the regions of the $\mathrm{DMN}$, such as the bilateral posterior cingulate gyrus, bilateral medial prefrontal cortex, bilateral hippocampus and parahippocampal gyrus, bilateral angular gyrus, and left lateral temporal cortex (left middle temporal gyrus [temporal pole part] and left inferior temporal gyrus). Additionally, this subnetwork featured connectivity of the left superior frontal gyrus (dorsolateral part) with the left parahippocampal gyrus and connectivity of the left superior frontal gyrus (dorsolateral part) with the left middle temporal gyrus (temporal pole part). Connectivity between the left middle frontal gyrus and left inferior temporal gyrus was also found in this subnetwork.

Meanwhile, in the second subnetwork, MHE patients showed a significant reduction of negative FC (Figure 2 and Table 3 ). This subnetwork predominantly involved connectivities between DMN regions (such as the bilateral posterior cingulate gyrus, medial prefrontal cortex, and angular gyrus) and the bilateral precentral and postcentral gyrus and bilateral Rolandic operculum. Additionally, connectivity of the right precentral gyrus with the left superior frontal gyrus (dorsolateral part) and connectivity of the right precentral gyrus with the left middle frontal gyrus were also found in this subnetwork.

In the patient group, Spearman correlation analysis revealed that the PHES score was significantly positively correlated with the strength of two FCs within the first subnetwork, namely, the connectivity between the left posterior cingulate gyrus and right hippocampus ( $r=0.556, P=0.044$, FDR-corrected) and the
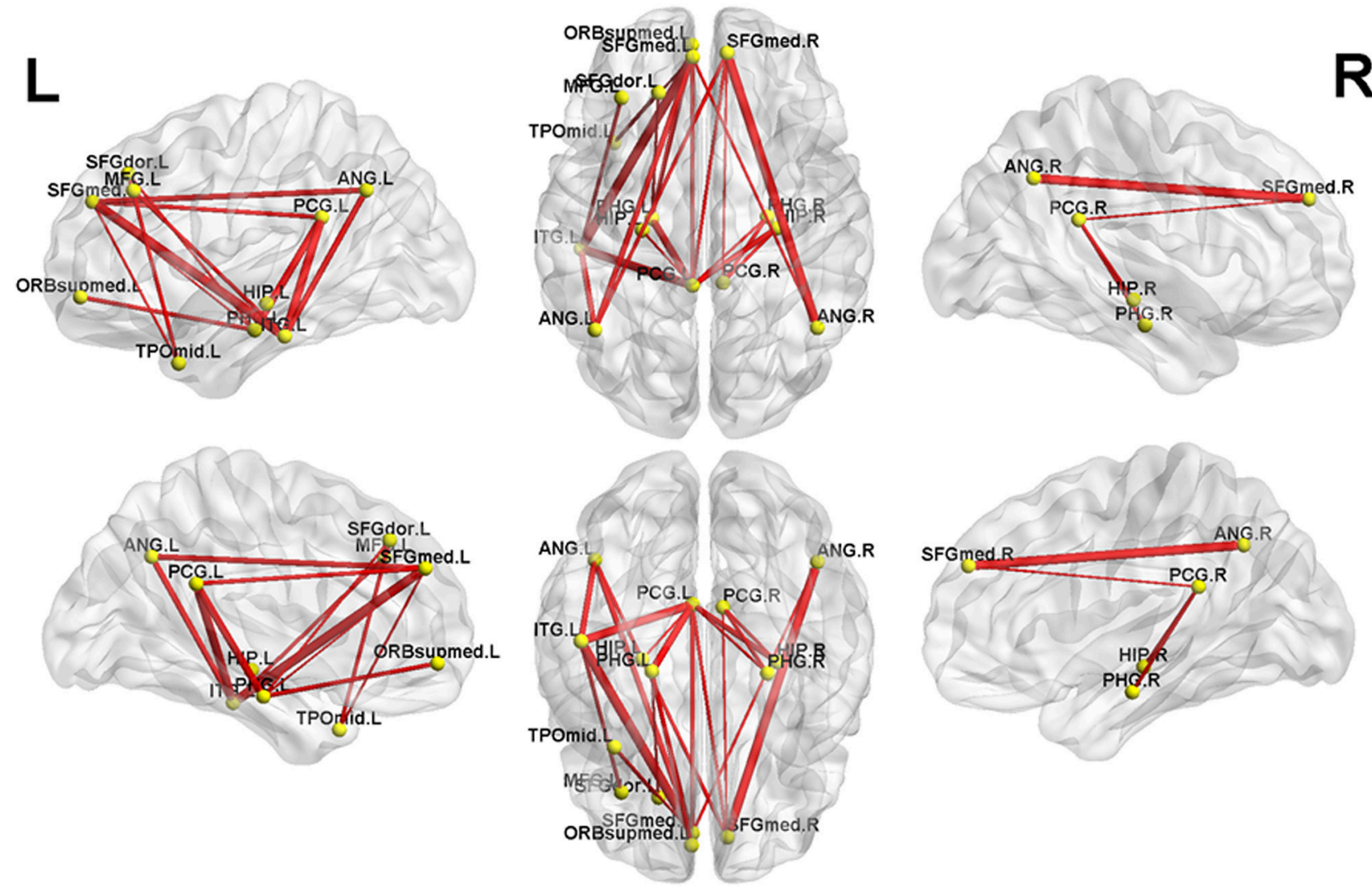

FIGURE 1 | The first subnetwork identified by network-based statistics. This subnetwork predominantly involves the regions of the default-mode network. The red line indicates the positive functional connectivity within this subnetwork. The edge size points out the significance of between-group difference in functional connectivity strength (see Table 2). SFGmed, superior frontal gyrus, medial; PCG, posterior cingulate gyrus; SFGdor, superior frontal gyrus, dorsolateral; HIP, hippocampus; PHG, parahippocampal gyrus; ORBsupmed, superior frontal gyrus, medial orbital; ANG, angular gyrus; TPOmid, temporal pole of middle temporal gyrus; MFG, middle frontal gyrus; ITG, inferior temporal gyrus. The letters "L" and "R" indicate left and right side, respectively. 
TABLE 2 | Functional connectivity within the first subnetwork identified by NBS analysis.

\begin{tabular}{|c|c|c|c|}
\hline \multirow[t]{2}{*}{ Connectivity } & \multicolumn{2}{|c|}{$\begin{array}{l}\text { Functional connectivity strength } \\
\text { (Pearson correlation coefficient) }\end{array}$} & \multirow[t]{2}{*}{$T$-value } \\
\hline & HC subjects & MHE patients & \\
\hline SFGmed.L to PCG.L & $0.58 \pm 0.15$ & $0.38 \pm 0.21$ & 3.55 \\
\hline SFGmed.L to PHG.L & $0.10 \pm 0.17$ & $-0.06 \pm 0.14$ & 3.10 \\
\hline SFGmed.L to ANG.L & $0.65 \pm 0.13$ & $0.41 \pm 0.24$ & 3.81 \\
\hline SFGmed.L to ANG.R & $0.32 \pm 0.20$ & $0.10 \pm 0.21$ & 3.24 \\
\hline SFGmed.L to TPOmid.L & $0.36 \pm 0.17$ & $0.17 \pm 0.19$ & 3.19 \\
\hline SFGmed.L to ITG.L & $0.27 \pm 0.16$ & $-0.01 \pm 0.21$ & 4.58 \\
\hline SFGmed.R to PCG.L & $0.54 \pm 0.19$ & $0.34 \pm 0.19$ & 3.34 \\
\hline SFGmed.R to PCG.R & $0.46 \pm 0.22$ & $0.25 \pm 0.19$ & 3.11 \\
\hline SFGmed.R to ANG.L & $0.55 \pm 0.18$ & $0.29 \pm 0.28$ & 3.43 \\
\hline SFGmed.R to ANG.R & $0.52 \pm 0.22$ & $0.22 \pm 0.19$ & 4.53 \\
\hline ORBsupmed.L to PHG.L & $0.31 \pm 0.18$ & $0.08 \pm 0.21$ & 3.55 \\
\hline PCG.L to HIP.L & $0.28 \pm 0.19$ & $0.04 \pm 0.26$ & 3.14 \\
\hline PCG.L to HIP.R & $0.24 \pm 0.18$ & $-0.01 \pm 0.23$ & 3.77 \\
\hline PCG.L to PHG.L & $0.25 \pm 0.16$ & $0.00 \pm 0.21$ & 4.13 \\
\hline PCG.L to PHG.R & $0.17 \pm 0.14$ & $-0.03 \pm 0.22$ & 3.20 \\
\hline PCG.L to ITG.L & $0.20 \pm 0.20$ & $-0.09 \pm 0.21$ & 4.38 \\
\hline PCG.R to HIP.R & $0.26 \pm 0.19$ & $0.05 \pm 0.21$ & 3.34 \\
\hline PCG.R to PHG.R & $0.23 \pm 0.14$ & $0.03 \pm 0.21$ & 3.41 \\
\hline ANG.L to ITG.L & $0.39 \pm 0.17$ & $0.14 \pm 0.23$ & 3.86 \\
\hline SFGdor.L to PHG.L & $0.04 \pm 0.18$ & $-0.14 \pm 0.17$ & 3.22 \\
\hline SFGdor.L to TPOmid.L & $0.17 \pm 0.17$ & $0.00 \pm 0.13$ & 3.46 \\
\hline MFG.L to ITG.L & $0.31 \pm 0.22$ & $0.06 \pm 0.23$ & 3.45 \\
\hline
\end{tabular}

SFGmed, superior frontal gyrus, medial; PCG, posterior cingulate gyrus; SFGdor, superior frontal gyrus, dorsolateral; HIP, hippocampus; PHG, parahippocampal gyrus; ORBsupmed, superior frontal gyrus, medial orbital; ANG, angular gyrus; TPOmid, temporal pole: middle temporal gyrus; MFG, middle frontal gyrus; ITG, inferior temporal gyrus. The letters " $L$ " and " $R$ " indicate left and right side, respectively.

connectivity between the left superior frontal gyrus (dorsolateral part) and left parahippocampal gyrus $(r=0.477, P=0.039$, uncorrected).

\section{DISCUSSION}

In the present study, we performed NBS analysis to identify FC changes related to MHE at the whole-brain functional connectome level for the first time. Network-wise FC changes were found in two brain subnetworks with significant FC reduction: One predominantly involved positive FC among DMN regions, including the bilateral posterior cingulate gyrus, bilateral medial prefrontal cortex, bilateral hippocampus and parahippocampal gyrus, bilateral angular gyrus, and left lateral temporal cortex; the other primarily involved negative FC among DMN regions (such as the bilateral posterior cingulate gyrus, medial prefrontal cortex, and angular gyrus) and regions that engage in the somatosensory network (i.e., bilateral precentral and postcentral gyri) and language network (i.e., bilateral Rolandic operculum). We also identified the correlations between FC within the default-mode subnetwork and MHE patients' cognitive performance. These findings provide new insights into the mechanisms of MHE.

The reduction in both positive and negative $\mathrm{FC}$ revealed by our study was consistent with the findings of previous whole-brain functional network analyses. In studies based on a seed region or conducted via pairwise coupling, MHE patients have always shown widespread FC abnormalities (predominantly decreased FC), especially in the DMN, attention network, and thalamocortical circuit $(8-10,25)$. Additionally, whole-brain functional connectome analyses using the graph theory-based method have revealed significant reductions of connectivity strength in MHE, which are predominantly located in bilateral midline brain areas and bilateral primary somatosensory and auditory regions (12). Notably, previous whole-brain FC analyses also found FC increases related to MHE in several regions involving the somatosensory network, visual network, and memory system, which is regarded as a compensatory mechanism $(8,9)$. We did not find such compensatory FC changes in the present study. There are two likely reasons for this discrepancy: differences in the samples (e.g., patients with heterogeneous etiologies of cirrhosis) and different data analysis methods with distinct sensitivity and pertinence.

The first brain subnetwork identified by NBS analysis in our study mainly included the DMN regions. This pattern is consistent with previous reports in which both static and dynamic resting-state FC showed disruption in the DMN (particularly in the medial prefrontal cortex and posterior cingulate gyrus) of patients with cirrhosis and MHE (26-29). These default-mode connectivity changes may be attributed to brain regional edema $(30,31)$ and impairment of the microstructural integrity of white matter fiber $(31,32)$. Furthermore, previous MHE studies also demonstrated a significant association between the altered connectivity strength within the DMN and clinical parameter (e.g., blood ammonia level and neuropsychological performance) abnormalities as well as the development and prognosis of HE disease (9, $26,29,31)$. In fact, it has been well-documented that DMN is involved in various cognitive processes, such as memory, attention, and executive functions (33-35). A study on MHE therapy demonstrated that the cognitive amelioration following treatment with oral L-ornithine is correlated with decreases in activation (i.e., increases in deactivation) in the posterior cingulate and ventral medial prefrontal cortex, two pivotal regions of the DMN (36). Thus, the correlations of the FC within the default-mode subnetwork (i.e., the connectivity between the left posterior cingulate gyrus and right hippocampus) and PHES results found this study were not surprising.

The second brain subnetwork identified by NBS analysis predominantly included the connectivity between DMN regions (such as the bilateral posterior cingulate gyrus, medial prefrontal cortex, and angular gyrus) and the bilateral precentral and postcentral gyri and bilateral Rolandic operculum. The bilateral precentral and postcentral gyri represent the primary somatosensory areas, in which the spontaneous neural activity and the energy metabolism are disturbed in MHE patients (3739). Meanwhile, the bilateral Rolandic operculum is one of the major regions involved in the language processing system, which 

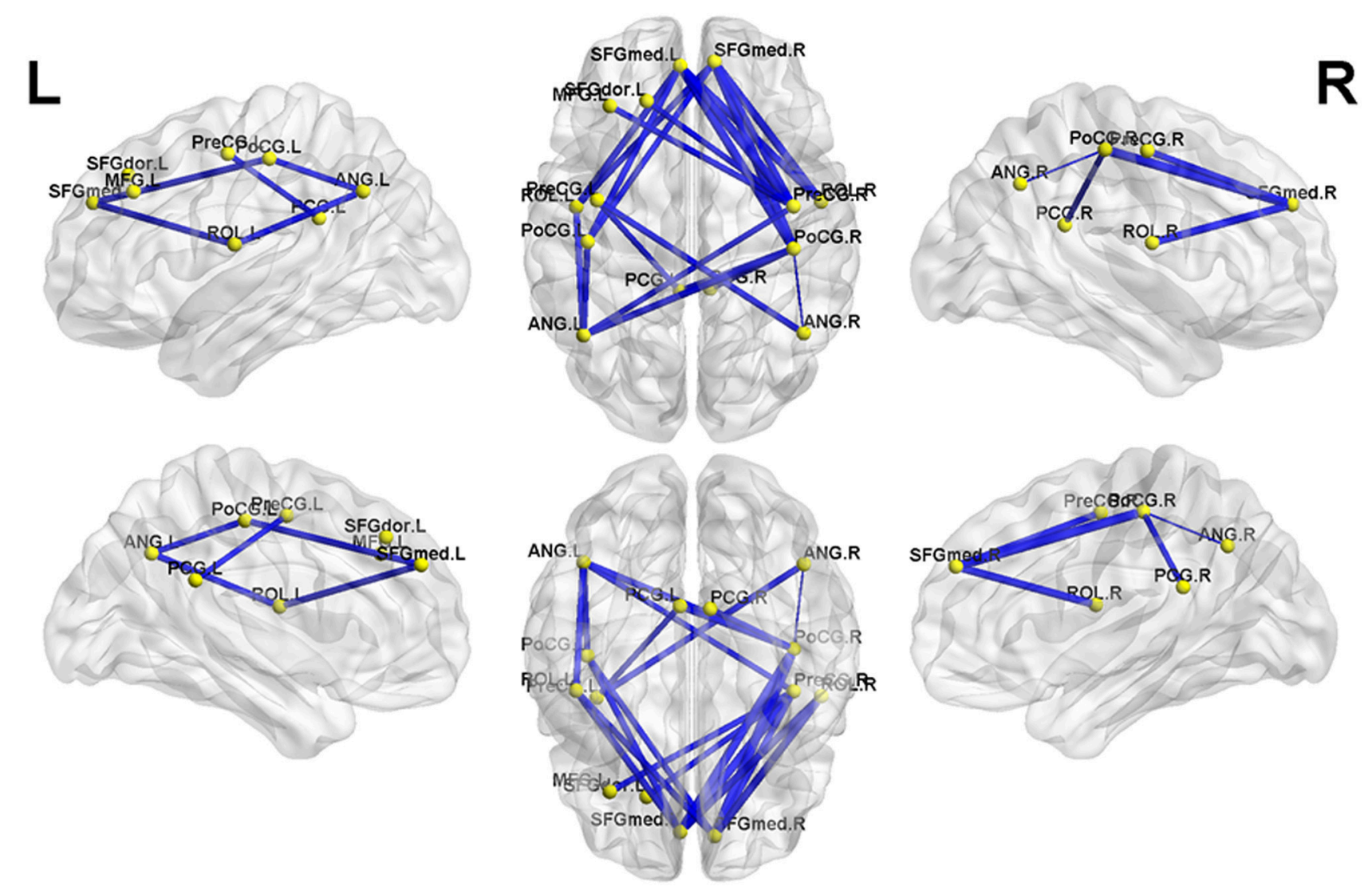

FIGURE 2 | The second subnetwork identified by network-based statistics. This subnetwork mainly involves the regions involved in the default-mode network, somatosensory network, and language network. The blue line indicates the negative functional connectivity within this subnetwork. The edge size points out the significance of between-group difference in functional connectivity strength (see Table 3). PreCG, precental gyrus; SFGdor, superior frontal gyrus, dorsolateral; MFG, middle frontal gyrus; SFGmed, superior frontal gyrus, medial; ROL, Rolandic operculum; PoCG, postcentral gyrus; PCG, posterior cingulate gyrus; ANG, angular gyrus. The letters " $L$ " and "R" indicate left and right side, respectively.

is especially associated with speech (40). Previous studies have revealed that MHE patients have decreased regional connectivity strength and efficiency in a few brain areas, including the bilateral Rolandic operculum $(11,12)$. Taken together, these existing reports suggest that MHE could induce dysfunction of the somatosensory and language systems, which is in line with our findings. In fact, neurophysiological and neuropsychological studies have demonstrated deficits in fine motor skills (such as extrapyramidal and cerebellar symptoms) in MHE patients $(41,42)$. Additionally, although language function is relatively spared in patients with cirrhosis and MHE, there have been some reports of language function decline in MHE (43). Of note, it has been well-documented that the proper FC pattern among the intrinsic brain networks is the key characteristic of human brain functional organization, which plays an important role in functional integration and is essential for various neural functions (44-46). Therefore, our finding that the negative connectivity between DMN regions and the areas of the somatosensory and language networks was decreased in MHE was in line with expectations. The neuropathology mechanisms underlying the FC reduction among these networks in $\mathrm{MHE}$ is not well understood and should be further addressed in the future.

We acknowledge several limitations of this work. First, this study had a relatively small sample size. Second, our design did not enable us to control for the potential confounding effects of heterogeneous etiologies of cirrhosis (e.g., the residual effect of past alcohol abuse). Previous studies have demonstrated that distinct etiologies could induce different degrees of brain structural and functional changes in cirrhotic patients $(47,48)$. Third, we could not evaluate whether network-wise FCs are related to deficits in specific cognitive domains (e.g., memory, attention, and executive domains), since only a general cognition assessment (i.e., the PHES examination) was performed. More comprehensive evaluation specific to the distinct cognitive domains is recommended for future studies. Fourth, the methodological limitations of NBS should be considered. NBS controls for multiple comparisons through cluster-based thresholding, but a widely acknowledged drawback of NBS is the arbitrary nature of threshold choice (14). To address this issue, we repeatedly performed NBS with a range of initial 
TABLE 3 | Functional connectivity within the second subnetwork identified by NBS analysis.

\begin{tabular}{|c|c|c|c|}
\hline \multirow[t]{2}{*}{ Connectivity } & \multicolumn{2}{|c|}{$\begin{array}{l}\text { Functional connectivity strength } \\
\text { (Pearson correlation coefficient) }\end{array}$} & \multirow[t]{2}{*}{$T$-value } \\
\hline & HC subjects & MHE patients & \\
\hline SFGmed.L to PreCG.R & $-0.43 \pm 0.16$ & $-0.15 \pm 0.20$ & 4.68 \\
\hline SFGmed.L to PoCG.L & $-0.39 \pm 0.18$ & $-0.13 \pm 0.26$ & 3.50 \\
\hline SFGmed.L to PoCG.R & $-0.48 \pm 0.18$ & $-0.15 \pm 0.24$ & 4.66 \\
\hline SFGmed.L to ROL.L & $-0.44 \pm 0.18$ & $-0.18 \pm 0.26$ & 3.60 \\
\hline SFGmed.L to ROL.R & $-0.47 \pm 0.18$ & $-0.18 \pm 0.27$ & 4.01 \\
\hline SFGmed.R to PreCG.R & $-0.42 \pm 0.20$ & $-0.18 \pm 0.22$ & 3.57 \\
\hline SFGmed.R to PoCG.L & $-0.40 \pm 0.19$ & $-0.17 \pm 0.23$ & 3.24 \\
\hline SFGmed.R to PoCG.R & $-0.50 \pm 0.17$ & $-0.16 \pm 0.23$ & 5.06 \\
\hline SFGmed.R to ROL.L & $-0.44 \pm 0.20$ & $-0.22 \pm 0.22$ & 3.33 \\
\hline SFGmed.R to ROL.R & $-0.46 \pm 0.18$ & $-0.19 \pm 0.21$ & 4.20 \\
\hline PCG.L to PreCG.L & $-0.30 \pm 0.18$ & $-0.10 \pm 0.23$ & 3.11 \\
\hline PCG.L to PoCG.R & $-0.35 \pm 0.18$ & $-0.13 \pm 0.20$ & 3.65 \\
\hline PCG.R to PoCG.R & $-0.34 \pm 0.23$ & $-0.13 \pm 0.18$ & 3.14 \\
\hline ANG.L to PreCG.R & $-0.44 \pm 0.17$ & $-0.23 \pm 0.24$ & 3.10 \\
\hline ANG.L to PoCG.L & $-0.40 \pm 0.18$ & $-0.11 \pm 0.34$ & 3.28 \\
\hline ANG.L to PoCG.R & $-0.47 \pm 0.17$ & $-0.22 \pm 0.24$ & 3.68 \\
\hline ANG.L to ROL.L & $-0.46 \pm 0.19$ & $-0.19 \pm 0.29$ & 3.48 \\
\hline ANG.R to PreCG.L & $-0.34 \pm 0.20$ & $-0.11 \pm 0.22$ & 3.45 \\
\hline ANG.R to PoCG.R & $-0.48 \pm 0.15$ & $-0.26 \pm 0.21$ & 3.53 \\
\hline SFGdor.L to PreCG.R & $-0.40 \pm 0.17$ & $-0.19 \pm 0.25$ & 3.12 \\
\hline MFG.L to PreCG.R & $-0.35 \pm 0.15$ & $-0.13 \pm 0.25$ & 3.35 \\
\hline
\end{tabular}

PreCG, precentral gyrus; SFGdor, superior frontal gyrus, dorsolateral; MFG, middle frontal gyrus; SFGmed, superior frontal gyrus, medial; ROL, Rolandic operculum; PoCG, postcentral gyrus; PCG, posterior cingulate gyrus; ANG, angular gyrus. The letters " $L$ " and " $R$ " indicate left and right side, respectively.

cluster-defining thresholds. The similarly of the results for the various thresholds validated the stability and reliability of our findings.

\section{REFERENCES}

1. Vilstrup H, Amodio P, Bajaj J, Cordoba J, Ferenci P, Mullen KD, et al. Hepatic encephalopathy in chronic liver disease: 2014 Practice Guideline by the American Association for the Study Of Liver Diseases and the European Association for the Study of the Liver. Hepatology (2014) 60:715-35. doi: 10.1002/hep.27210

2. Ferenci P, Lockwood A, Mullen K, Tarter R, Weissenborn K, Blei AT. Hepatic encephalopathy-definition, nomenclature, diagnosis, and quantification: final report of the working party at the 11th World Congresses of Gastroenterology, Vienna, 1998. Hepatology (2002) 35:716-21. doi: 10.1053/jhep.2002. 31250

3. Bajaj JS, Wade JB, Sanyal AJ. Spectrum of neurocognitive impairment in cirrhosis: implications for the assessment of hepatic encephalopathy. Hepatology (2009) 50:2014-21. doi: 10.1002/hep.23216

4. Bajaj JS, Saeian K, Schubert CM, Hafeezullah M, Franco J, Varma RR, et al. Minimal hepatic encephalopathy is associated with motor vehicle crashes: the reality beyond the driving test. Hepatology (2009) 50:1175-83. doi: 10.1002/hep.23128

5. Dhiman RK, Kurmi R, Thumburu KK, Venkataramarao SH, Agarwal R, Duseja A, et al. Diagnosis and prognostic significance of minimal hepatic encephalopathy in patients with cirrhosis of liver. Dig Dis Sci. (2010) 55:238190. doi: 10.1007/s10620-010-1249-7
In summary, our findings indicate that DMN dysfunction may be one of the core issues in the pathophysiology of MHE. The decreased connectivity within the DMN and between the $\mathrm{DMN}$ and several networks, such as somatosensory and language networks, is the consistent and key characteristic of early $\mathrm{HE}$ and may be responsible for the mechanisms underlying MHE. Our results provide further support for the notion that $\mathrm{HE}$ is a neurological disease related to intrinsic brain network disruption.

\section{AUTHOR CONTRIBUTIONS}

$\mathrm{H}-\mathrm{JC}$ and T-XZ conceived and designed the study, acquired and analyzed the data, and wrote the manuscript. CZ and Y-QG contributed to data analysis. All authors have read and approved the manuscript.

\section{FUNDING}

This study was funded by the grants from the National Natural Science Foundation of China (No. 81501450), Fujian Provincial Science Fund for Distinguished Young Scholars (No. 2018J06023), Fujian Provincial Program for Distinguished Young Scholars (No. 2017B023), and Fujian Provincial Health Commission Project for Scientific Research Talents (No. 2018ZQN-28).

\section{SUPPLEMENTARY MATERIAL}

The Supplementary Material for this article can be found online at: https://www.frontiersin.org/articles/10.3389/fneur. 2019.00033/full\#supplementary-material

Supplementary Figure 1 | The functional connectivity pattern of the subnetwork identified by network-based statistics with different initial cluster-defining thresholds $(t=2.8-3.4)$. Very similar results were obtained across these analyses.
6. Ortiz M, Jacas C, Cordoba J. Minimal hepatic encephalopathy: diagnosis, clinical significance and recommendations. J. Hepatol. (2005) 42(Suppl):S4553. doi: 10.1016/j.jhep.2004.11.028

7. Prasad S, Dhiman RK, Duseja A, Chawla YK, Sharma A, Agarwal R. Lactulose improves cognitive functions and health-related quality of life in patients with cirrhosis who have minimal hepatic encephalopathy. Hepatology (2007) 45:549-59. doi: 10.1002/hep.21533

8. Zhang LJ, Zheng G, Zhang L, Zhong J, Wu S, Qi R, et al. Altered brain functional connectivity in patients with cirrhosis and minimal hepatic encephalopathy: a functional MR imaging study. Radiology (2012) 265:52836. doi: 10.1148/radiol.12120185

9. Chen HJ, Jiang LF, Sun T, Liu J, Chen QF, Shi HB. Resting-state functional connectivity abnormalities correlate with psychometric hepatic encephalopathy score in cirrhosis. Eur J Radiol. (2015) 84:2287-95. doi: 10.1016/j.ejrad.2015.08.005

10. Zhang G, Cheng Y, Liu B. Abnormalities of voxel-based wholebrain functional connectivity patterns predict the progression of hepatic encephalopathy. Brain Imaging Behav. (2017) 11:784-96. doi: 10.1007/s11682-016-9553-2

11. Hsu TW, Wu CW, Cheng YF, Chen HL, Lu CH, Cho KH, et al. Impaired small-world network efficiency and dynamic functional distribution in patients with cirrhosis. PLoS ONE (2012) 7:e35266. doi: 10.1371/journal.pone.0035266 
12. Jao T, Schroter M, Chen CL, Cheng YF, Lo CY, Chou KH, et al. Functional brain network changes associated with clinical and biochemical measures of the severity of hepatic encephalopathy. Neuroimage (2015) 122:332-44. doi: 10.1016/j.neuroimage.2015.07.068

13. Zheng G, Zhang L, Zhang LJ, Li Q, Pan Z, Liang X, et al. Altered modular organization of functional connectivity networks in cirrhotic patients without overt hepatic encephalopathy. Biomed Res Int. (2014) 2014:727452. doi: 10.1155/2014/727452

14. Zalesky A, Fornito A, Bullmore ET. Network-based statistic: identifying differences in brain networks. Neuroimage (2010) 53:1197-207. doi: 10.1016/j.neuroimage.2010.06.041

15. Zajac L, Koo BB, Bauer CM, Killiany R, Behalf Of The Alzheimer's Disease Neuroimaging I. Seed location impacts whole-brain structural network comparisons between healthy elderly and individuals with Alzheimer's disease. Brain Sci. (2017) 7:37. doi: 10.3390/brainsci70 40037

16. Lopes R, Delmaire C, Defebvre L, Moonen AJ, Duits AA, Hofman P, et al. Cognitive phenotypes in parkinson's disease differ in terms of brainnetwork organization and connectivity. Hum Brain Mapp. (2017) 38:1604-21. doi: 10.1002/hbm.23474

17. McNabb CB, Tait RJ, McIlwain ME, Anderson VM, Suckling J, Kydd RR, et al. Functional network dysconnectivity as a biomarker of treatment resistance in schizophrenia. Schizophr Res. (2018) 195:160-7. doi: 10.1016/j.schres.2017.10.015

18. Pua EPK, Malpas CB, Bowden SC, Seal ML. Different brain networks underlying intelligence in autism spectrum disorders. Hum Brain Mapp. (2018) 39:3253-62. doi: 10.1002/hbm.24074

19. Lai CH, Wu YT, Hou YM. Functional network-based statistics in depression: theory of mind subnetwork and importance of parietal region. J Affect Disord. (2017) 217:132-7. doi: 10.1016/j.jad.2017.03.073

20. Cui Y, Li SF, Gu H, Hu YZ, Liang X, Lu CQ, et al. Disrupted brain connectivity patterns in patients with type 2 diabetes. AJNR Am J Neuroradiol. (2016) 37:2115-22. doi: 10.3174/ajnr.A4858

21. Ji L, Zhang H, Potter GG, Zang YF, Steffens DC, Guo H, et al. Multiple neuroimaging measures for examining exercise-induced neuroplasticity in older adults: a quasi-experimental study. Front Aging Neurosci. (2017) 9:102. doi: 10.3389/fnagi.2017.00102

22. Bullmore E, Sporns O. Complex brain networks: graph theoretical analysis of structural and functional systems. Nat Rev Neurosci. (2009) 10:186-98. doi: $10.1038 / \mathrm{nrn} 2575$

23. Smith SM, Vidaurre D, Beckmann CF, Glasser MF, Jenkinson M, Miller KL, et al. Functional connectomics from resting-state fMRI. Trends Cogn Sci. (2013) 17:666-82. doi: 10.1016/j.tics.2013.09.016

24. Yang FC, Chou KH, Hsu AL, Fuh JL, Lirng JF, Kao HW, et al. Altered brain functional connectome in migraine with and without restless legs syndrome: a resting-state functional MRI study. Front Neurol. (2018) 9:25. doi: 10.3389/fneur.2018.00025

25. Lv XF, Wu HW, Tian L, Han LJ, Li J, Qiu YW, et al. Aberrant restingstate functional connectivity density in patients with hepatitis B virusrelated cirrhosis. Biomed Res Int. (2016) 2016:4168512. doi: 10.1155/2016/41 68512

26. Chen HJ, Jiao Y, Zhu XQ, Zhang HY, Liu JC, Wen S, et al. Brain dysfunction primarily related to previous overt hepatic encephalopathy compared with minimal hepatic encephalopathy: resting-state functional MR imaging demonstration. Radiology (2013) 266:261-70. doi: 10.1148/radiol.12120026

27. Chen HJ, Lin HL, Chen QF, Liu PF. Altered dynamic functional connectivity in the default mode network in patients with cirrhosis and minimal hepatic encephalopathy. Neuroradiology (2017) 59:905-14. doi: 10.1007/s00234-017-1881-4

28. Garcia-Garcia R, Cruz-Gomez AJ, Mangas-Losada A, Urios A, Forn C, Escudero-Garcia D, et al. Reduced resting state connectivity and gray matter volume correlate with cognitive impairment in minimal hepatic encephalopathy. PLoS ONE (2017) 12:e0186463. doi: 10.1371/journal.pone.0186463

29. Qi R, Zhang LJ, Xu Q, Liang X, Luo S, Zhang Z, et al. Abnormal functional connectivity within the default mode network in patients with HBVrelated cirrhosis without hepatic encephalopathy revealed by resting-state functional MRI. Brain Res. (2014) 1576:73-80. doi: 10.1016/j.brainres.2014 05.044

30. Lin WC, Hsu TW, Chen CL, Wu CW, Lu CH, Chen HL, et al. Connectivity of default-mode network is associated with cerebral edema in hepatic encephalopathy. PLoS ONE (2012) 7:e36986. doi: 10.1371/journal.pone.0036986

31. Qi R, Xu Q, Zhang LJ, Zhong J, Zheng G, Wu S, et al. Structural and functional abnormalities of default mode network in minimal hepatic encephalopathy: a study combining DTI and fMRI. PLoS ONE (2012) 7:e41376. doi: 10.1371/journal.pone.00 41376

32. Chen HJ, Wang Y, Yang M, Zhu XQ, Teng GJ. Aberrant interhemispheric functional coordination in patients with HBV-related cirrhosis and minimal hepatic encephalopathy. Metab Brain Dis. (2014) 29:617-23. doi: 10.1007/s11011-014-9505-8

33. Fox MD, Snyder AZ, Vincent JL, Corbetta M, Van Essen DC, Raichle ME The human brain is intrinsically organized into dynamic, anticorrelated functional networks. Proc Natl Acad Sci USA. (2005) 102:9673-8. doi: 10.1073/pnas.0504136102

34. Menon V. Large-scale brain networks and psychopathology: a unifying triple network model. Trends Cogn Sci. (2011) 15:483-506. doi: 10.1016/j.tics.2011.08.003

35. Raichle ME, MacLeod AM, Snyder AZ, Powers WJ, Gusnard DA, Shulman GL. A default mode of brain function. Proc Natl Acad Sci USA. (2001) 98:676-82. doi: 10.1073/pnas.98.2.676

36. McPhail MJ, Leech R, Grover VP, Fitzpatrick JA, Dhanjal NS, Crossey MM, et al. Modulation of neural activation following treatment of hepatic encephalopathy. Neurology (2013) 80:1041-7. doi: 10.1212/WNL.0b013e31828726e1

37. Iversen P, Sorensen M, Bak LK, Waagepetersen HS, Vafaee MS, Borghammer P, et al. Low cerebral oxygen consumption and blood flow in patients with cirrhosis and an acute episode of hepatic encephalopathy. Gastroenterology (2009) 136:863-71. doi: 10.1053/j.gastro.2008 10.057

38. Lv XF, Qiu YW, Tian JZ, Xie CM, Han LJ, Su HH, et al. Abnormal regional homogeneity of resting-state brain activity in patients with HBV-related cirrhosis without overt hepatic encephalopathy. Liver Int. (2013) 33:375-83. doi: 10.1111/liv.12096

39. Lv XF, Ye M, Han LJ, Zhang XL, Cai PQ, Jiang GH, et al. Abnormal baseline brain activity in patients with HBV-related cirrhosis without overt hepatic encephalopathy revealed by resting-state functional MRI. Metab Brain Dis. (2013) 28:485-92. doi: 10.1007/s11011-0139420-4

40. Indefrey $\mathrm{P}$, Brown $\mathrm{CM}$, Hellwig $\mathrm{F}$, Amunts $\mathrm{K}$, Herzog $\mathrm{H}$, Seitz RJ, et al. A neural correlate of syntactic encoding during speech production. Proc Natl Acad Sci USA. (2001) 98:5933-6. doi: 10.1073/pnas.1011 18098

41. Butz M, Timmermann L, Braun M, Groiss SJ, Wojtecki L, Ostrowski $S$, et al. Motor impairment in liver cirrhosis without and with minimal hepatic encephalopathy. Acta Neurol Scand. (2010) 122:27-35. doi: 10.1111/j.1600-0404.2009.01246.x

42. Tryc AB, Goldbecker A, Berding G, Rumke S, Afshar K, Shahrezaei $\mathrm{GH}$, et al. Cirrhosis-related Parkinsonism: prevalence, mechanisms and response to treatments. J Hepatol. (2013) 58:698-705. doi: 10.1016/j.jhep.2012. 11.043

43. Adekanle O, Sunmonu TA, Komolafe MA, Ndububa DA. Cognitive functions in patients with liver cirrhosis: assessment using community screening interview for dementia. Ann Afr Med. (2012) 11:222-9. doi: 10.4103/1596-3519.102853

44. Allen EA, Erhardt EB, Damaraju E, Gruner W, Segall JM, Silva RF, et al. A baseline for the multivariate comparison of resting-state networks. Front Syst Neurosci. (2011) 5:2. doi: 10.3389/fnsys.2011. 00002

45. Faghiri A, Stephen JM, Wang YP, Wilson TW, Calhoun VD. Changing brain connectivity dynamics: from early childhood to adulthood. Hum Brain Mapp. (2018) 39:1108-17. doi: 10.1002/hbm. 23896 
46. Jafri MJ, Pearlson GD, Stevens M, Calhoun VD. A method for functional network connectivity among spatially independent restingstate components in schizophrenia. Neuroimage (2008) 39:1666-81. doi: 10.1016/j.neuroimage.2007.11.001

47. Guevara M, Baccaro ME, Gomez-Anson B, Frisoni G, Testa C, Torre A, et al. Cerebral magnetic resonance imaging reveals marked abnormalities of brain tissue density in patients with cirrhosis without overt hepatic encephalopathy. J Hepatol. (2011) 55:564-73. doi: 10.1016/j.jhep.2010.12.008

48. Lee Y, Kim C, Suk KT, Choi HC, Bang CS, Yoon JH, et al. Differences in cognitive function between patients with viral and alcoholic compensated liver cirrhosis. Metab Brain Dis. (2016) 31:369-76. doi: 10.1007/s11011-015-9761-2
Conflict of Interest Statement: The authors declare that the research was conducted in the absence of any commercial or financial relationships that could be construed as a potential conflict of interest.

Copyright (c) 2019 Zhan, Chen, Gao and Zou. This is an open-access article distributed under the terms of the Creative Commons Attribution License (CC BY). The use, distribution or reproduction in other forums is permitted, provided the original author(s) and the copyright owner(s) are credited and that the original publication in this journal is cited, in accordance with accepted academic practice. No use, distribution or reproduction is permitted which does not comply with these terms. 